\title{
Automaticity and Reestablishment of Executive Control-An fMRI Study
}

\author{
Andrea Kübler ${ }^{1,2}$, Veronica Dixon ${ }^{3}$, and Hugh Garavan ${ }^{1,3}$
}

\begin{abstract}
The ability to exert control over automatic behavior is of particular importance as it allows us to interrupt our behavior when the automatic response is no longer adequate or even dangerous. However, despite the literature that exists on the effects of practice on brain activation, little is known about the neuroanatomy involved in reestablishing executive control over previously automatized behavior. We present a visual search task that enabled participants to automatize according to defined criteria within about $3 \mathrm{hr}$ of practice and then
\end{abstract}

\section{INTRODUCTION}

In many regards, automatic or habitual behavior is the opposite to executively controlled behavior by virtue of being stimulus driven, ballistic, and often unintended. According to Logan's instance theory of automaticity (Compton \& Logan, 1991; Logan, 1988, 2002) each experience with a stimulus lays down a separate trace in memory such that automaticity is strengthened with every encounter of the stimulus. This theory further assumes that encoding into and retrieval from memory are unavoidable consequences of attention and that performance is regarded as automatic if behavior is guided by direct memory retrieval. Novices' performance on a particular task is based on computing an algorithm suitable to achieve the desired result. The more often novices are confronted with the same problem, the more likely it is that they will retrieve the solution from memory and will eventually abandon the algorithm entirely. Thus, automatization is characterized by the transition from algorithm-based to memory-based processing with a speedup in processing and reduced variability being the major quantitative behavioral properties of automatization (Logan, 1988). To enable the transition to memory-based processing, automaticity of a particular behavior can only be achieved when the same stimuli require the same responses throughout practice, a process referred to as consistent mapping (Schneider \& Shiffrin, 1977). Transfer to novel stimuli is poor

\footnotetext{
${ }^{1}$ Trinity College Dublin, Ireland, ${ }^{2}$ University of Tübingen, Germany, ${ }^{3}$ Medical College of Wisconsin
}

required them to reassert control without changing the stimulus set. We found widespread cortical activation early in practice. Activation in all frontal areas and in the inferior parietal lobule decreased significantly with practice. Only selected prefrontal (Brodmann's areas [BAs] 9/46/8) and parietal areas (BAs 39/40) were specifically reactivated when executive control was required, underlining the crucial role of the dorsolateral prefrontal cortex in executive control to guide our behavior.
(Logan, 1988, 2002), but is facilitated by stimulus similarity (Palmeri, 1997).

When task rules change within the same stimulus set it is necessary to reestablish executive control over an automatic process. This reestablishment requires a number of executive functions such as the inhibition of the prepotent response tendency and increased endogenous attentional control to apply the new task rule. However, once automatic behavior is established, it is typically quite difficult to reestablish executive control over performance, as the speed of the automatic process tends to produce a faster response than the deliberative, algorithmic process. This characteristic is quite apparent phenomenologically in the Stroop task (Stroop, 1935), in which the faster, automatic word-naming operation tends to interfere with the slower color naming despite the subject's best (executive) intentions.

A large body of functional imaging studies of practice effects exists (Kelly \& Garavan, 2005). Neuroanatomically, practice tends to either increase or decrease activation within the involved cortical network or produces a functional reorganization, indicating that the cortical regions recruited for task processing are altered with practice. Activation decreases are most often seen in complex sensorimotor or working memory tasks that do not involve a change in the underlying cognitive operations. This decrease in activation in the cortical network is interpreted as increased neural efficiency in task processing (Beauchamp, Dagher, Aston, \& Doyon, 2003; Toni, Rowe, Stephan, \& Passingham, 2002; Buchel, Coull, \& Friston, 1999). In motor skill learning, a pattern of increasing and decreasing activation can be observed 
such that activation decreases with practice in control and attentional areas located in the prefrontal and parietal cortex, whereas in task-specific areas of the premotor and motor cortex and cerebellum activation increases (Debaere, Wenderoth, Sunaert, Van Hecke, \& Swinnen, 2004; Frutiger et al., 2000). This redistribution (Kelly \& Garavan, 2005) of activation is in accordance with explicit attention to performance when confronted with a novel task that is replaced with practice by implicit or procedural task performance. Altered functional activations underlying task performance are most often seen in cognitive tasks that imply different cognitive processes operating early and late in practice (Poldrack \& Gabrieli, 2001; Raichle et al., 1994). For example, mirror reading requires visuospatial transformation early in learning that is replaced by word recognition later in practice.

The extensive literature on practice effects stands in contrast to the sparse research into the neuroanatomy through which executive control is asserted over a highly practiced task. The few studies available either presented their participants with novel stimuli (Raichle et al., 1994) or required them to reattend to the performance of an overlearned task (Jueptner et al., 1997). In both of these cases, prefrontal areas involved in attentional control were reactivated. To our knowledge, the dynamics involved in reasserting control over a behavior that is demonstrably automatic have not been addressed. These dynamics are quite relevant for clinical conditions in which overcoming habitual behaviors is difficult, such as addiction, obsessive-compulsive disorder, or the ingrained behavioral and cognitive patterns evident in depressed patients. Furthermore, exerting control over otherwise automatic behaviors occurs frequently in everyday life as experienced by drivers when traffic conditions suddenly worsen or by the effort involved in not putting sugar in one's coffee when dieting. To investigate these processes, we report data from a visual search task (VST) that (a) requires attentional control early in practice but becomes automated with time, (b) provides behavioral measures of the transition from controlled to automatic performance, (c) can be changed by instruction alone (i.e., without changing the stimuli or response demands) to require subjects to reassert control over their automatic behavior, and (d) can all be completed within a reasonable number of hours in the same day to facilitate functional imaging.

In Experiment 1, we established the VST (Figure 1) and showed with a sample of 16 participants that people can automatize within about $3 \mathrm{hr}$ of practice. Participants were trained on the task on five subsequent days always at the same time of day. The VST contained different set sizes, and participants had to indicate whether a target was present or not. We postulated that at the beginning of training participants would use a slow serial search strategy to detect the target, whereas

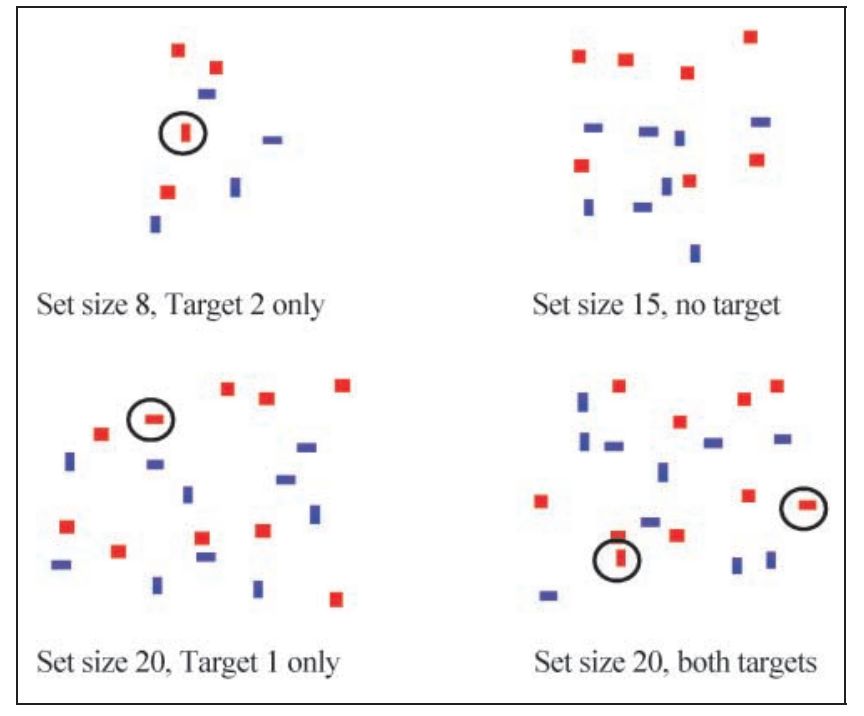

Figure 1. Examples of sets in the VST with 8, 15, or 20 items in the set. Before the switch the target was a red horizontal rectangle (Target 1), and after the switch, a red vertical rectangle (Target 2). Targets are encircled. Sets contained either both targets, Target 1 or 2 only, or no target. Sets were the same throughout the task.

with practice they would recall target and nontarget patterns from memory (Klapp, Boches, Trabert, \& Logan, 1991; Logan \& Klapp, 1991). Accordingly, the development of automaticity would be demonstrated by asymptotic reaction time and the disappearance of a stimulus set size effect. Following a change in target (further referred to as a switch), participants had to recommence the serial search strategy and overcome the learned stimulus-driven, memory-based response, which we expected to lead to increased reaction time and a reemergence of the set size effect. The presence of the two targets in the trials was balanced such that a simple response reversal would not suffice. With a sample of 72 participants, we investigated in Experiment 2 if similar results would be obtained within one day of practice. This was done with respect to functional magnetic resonance imaging (fMRI) requirements. Finally, in Experiment 3 we used the task to investigate the neural network underlying automaticity and reestablishment of executive control over previously automatized behavior. With a sample of 24 participants, functional imaging was conducted early in practice, late in practice, and during the target switch.

\section{METHODS}

\section{The Task}

Participants were presented with sets of blue rectangles, red squares, and red targets on a white background (Figure 1). Rectangles and squares were assigned to random positions within a circle (which was not shown on the screen) of $r=10 \mathrm{~cm}$; the items did not overlap. Sets contained 8, 15, or 20 items. Target 1 was a red 
horizontal rectangle, Target 2 a red vertical rectangle (identified by circles in Figure 1). Sets contained both targets, Target 1 only, Target 2 only, or no targets. These different trial types ensured that after the target switch a simple reversal of the response strategy would not suffice. For each set size and trial type, two different patterns were designed. Sets were presented for $1 \mathrm{sec}$ in the first block and for $800 \mathrm{msec}$ in all following blocks. Participants were required to press the left mouse key whenever they detected Target 1 in the set and the right mouse key if Target 1 was not present. They were instructed to be as accurate as possible, but at the same time to respond as fast as they could. Feedback (correct, incorrect) was given after each trial. If participants did not respond within $1 \mathrm{sec}$ (or $800 \mathrm{msec}$ for all blocks after Block 1) the feedback was "no response detectedplease try to respond faster." Before the start of a new run of trials, the word PREPARE appeared on the screen to allow participants to get ready. After practice, participants were instructed to search for Target 2 in the sets. They were now required to press the left mouse key when Target 2 was present and the right mouse key when it was not present. The point in time where participants were required to search for the new target within the well-known sets is referred to as "switch." No mention was made of Target 2 to participants prior to the switch.

Altogether, 112 participants took part in the study, which was approved by the institutional review board of the Medical College of Wisconsin and the ethics committee of the Department of Psychology, Trinity College, Dublin.

\section{Experiment 1}

Sixteen participants ( 4 men, mean age $\pm S D: 20.38 \pm$ 2.68 years, age range: 18-28 years) performed five practice blocks on five consecutive days, always at the same time of the day. Blocks comprised six runs of 1152 trials (totaling 5760 trials) and lasted about $30 \mathrm{~min}$, which were separated by 30 -sec breaks. Participants were presented with two different stimulus patterns per set size $(8,15$, or 20 items) and trial type (Target 1 only, Target 2 only, both targets present, neither target present) and each set was presented eight times per run resulting in 192 trials per run. On Day 4, participants were presented with two runs of the task as described. Thereafter, they were instructed to search for Target 2.

\section{Data Analysis \\ Individual Participants}

To assess automaticity, reaction time as a function of runs and set size was calculated for correct trials only. Participants were regarded as automatized when no main effect of set size and no differences in reaction time $(p>$
.10) were found in three consecutive runs (two-way univariate analysis of variance [ANOVA] with set size (3) and runs (3) as factors). In all experiments, analyses were restricted to just those participants who automatized.

\section{Group Data}

Repeated measures ANOVAs with set size or with set size and runs as factors were applied to the group data. All post hoc pairwise comparisons were corrected according to Holm (1979).

\section{Experiment 2}

To adapt the VST to scanning requirements, we reduced the stimulus patterns per set size, decreased the number of trials (3456 presented within 36 runs separated into six blocks), and reduced practice to $2.5 \mathrm{hr}$ on one day. Seventy-two participants (28 men, mean age $\pm S D$ : $22.74 \pm 7.77$ years, age range: $18-73$ years) performed the shorter version of the VST within one practice session. Participants were presented with only one stimulus pattern per set size and trial type with each presented eight times per run leading to 96 trials per run. Participants were presented with six blocks with each block comprised of six runs separated by 30-sec breaks. Blocks were separated by 5- or 10-min breaks.

\section{Experiment 3}

The same VST as in Experiment 2 was used. For scanning reasons the number of trials per run was increased to 108 (number of practice runs was shortened to 23). Thus, each set size and trial type was presented 9 times per run. The number of runs after the switch was reduced to 4 (instead of 10) to shorten the total time for the experiment (2916 trials presented in 27 runs). Note that the number of practice trials before the switch remained the same as in Experiment 2 (2496 in Experiment 2 and 2484 in Experiment 3; the difference of 12 trials was due to scanning constraints). Twenty-four participants ( 7 men, mean age $\pm S D: 29.42 \pm 8.66$ years, age range: 18-47 years) were imaged at the beginning of training for 6 runs (648 trials). Thereafter, they practiced three blocks of 5 runs (1620 trials) each outside the scanner. During the second scan, the instruction for the target switch was presented after 2 runs, and 4 further runs were completed after the switch (second scanning 648 trials).

\section{Functional Magnetic Resonance Imaging}

Nineteen contiguous $7-\mathrm{mm}$ sagittal slices covering the entire brain were collected using a blipped gradientecho, echo-planar pulse sequence $(\mathrm{TE}=40 \mathrm{msec}$; $\mathrm{TR}=$ 2000 msec; FOV $=24 \mathrm{~cm} ; 64 \times 64$ matrix; $3.75 \times$ $3.75 \mathrm{~mm}$ in-plane resolution). All scanning was conducted on a $1.5 \mathrm{~T}$ GE Signa scanner equipped with a 
30.5-cm i.d., three-axis local gradient coil, and an endcapped quadrature birdcage radio-frequency head coil (Wong, Boskamp, \& Hyde, 1992). For each participant, two high-resolution (SPGR) anatomic images were acquired prior to functional imaging (first and second scanning) to allow subsequent anatomical localization and normalization of functional activation. Stimuli were back-projected onto a screen at the subject's feet and were viewed with the aid of prism glasses attached to the inside of the radio-frequency head coil.

\section{Functional Analysis}

All data processing was conducted with the software package AFNI (Cox, 1996; Medical College of Wisconsin). Time shifting, using Fourier interpolation to adjust for differences in slice acquisition times, 3-D motion correction, and edge detection algorithms were first applied to the functional data. One scan comprised 216 trials (two runs) and three scans were recorded early in practice. A scan consisted of six on-off blocks; that is, 36 trials were followed by a 30-sec rest period. After practice outside the scanner, one scan preswitch and two scans postswitch were recorded. The average percentage change in signal for the six "on periods" of each scan was calculated relative to the average signal during the rest periods. These percent change scores served as the basic unit of analysis and are subsequently referred to as "activation." Activation maps were converted to a standard stereotaxic coordinate system (Talairach \& Tourneaux, 1988) and spatially blurred using a 4.2-mm full width at half-maximum isotropic Gaussian filter. Monte Carlo simulations revealed that a voxelwise threshold $(p=.001)$ combined with a minimum cluster size criterion $(174 \mu \mathrm{l})$ resulted in a posterior voxelwise threshold of $p=10^{-6}$ and a $p<.01$ false positive level for a cluster of activation. This threshold was used for all subsequent $t$ tests. Basic task activation maps for each scan were identified with one-sample $t$ tests against the null hypotheses of no change in activation. Following previous studies, activation maps for all scans were combined (OR map) such that voxels were included if significant in any one scan (Hester et al., 2005; Kübler, Murphy, \& Garavan, 2005; Kaufman, Ross, Stein, \& Garavan, 2003; Kübler, Murphy, Kaufman, Stein, \& Garavan, 2003). This is a more inclusive analysis in which significant activation from all scans are taken into account with no bias of the regions of interest (ROIs) toward a specific task section (early or late in practice). Brodmann's area (BA) templates, as provided by AFNI, were applied to the OR map identifying significantly activated voxels within each BA. The resulting clusters were used as functionally and anatomically defined ROIs and activation was averaged over all the voxels of a cluster and over clusters within each BA resulting in 37 ROIs. For each ROI, activation at the beginning of practice (Scan 1), preswitch (Scan 4), and postswitch (Scan 5) was compared using a repeated measures ANOVA with scans as within-subject factors; post hoc pairwise comparisons were corrected according to Holm (1979). To ensure that averaging over clusters within a BA did not obscure effects that might be present in just one cluster, the repeated measures ANOVA was performed on each of the 96 individual clusters that were included $>100 \mu$ of activated voxels. ${ }^{1}$ This analysis yielded identical results with two exceptions. One cluster in BA 8 and one cluster in BA 40 showed increased activation from pre- to postswitch and these clusters are listed separately in Table 1. Finally, bilateral clusters within each BA were also tested for hemispheric differences.

\section{RESULTS \\ Experiment 1}

Five participants did not automatize, and from another, an entire block was lost, leaving 10 participants for analyses. A repeated measures ANOVA with runs (first run of practice, last run before the switch, and first run after the switch) and set size (8, 15, 20) as withinsubjects factor found a main effect of run, $F(2,18)=$ $237.8, p<.001$; set size, $F(2,18)=42.9, p<.001$; and a significant interaction, $F(4,36)=15.6, p<.001)$. Reaction time difference between set sizes 8,15 , and 20 were all significant $(p<.05)$. With practice, the set size effect disappeared and reaction time became asymptotic (Figure $2 \mathrm{~A}$ ). Before the instruction to search for a new target, reaction time was the same for all set sizes (all pairwise comparisons nonsignificant). After the switch, reaction time increased and the set size effect reappeared (all pairwise comparisons $p<.05$ ). Reaction time after the switch was faster than at the beginning of training. It is important to note that the set size effect postswitch remained significant throughout the final 10 runs after the target switch (repeated measures ANOVA with set size as independent variable calculated for each run; all pairwise comparisons between set sizes within runs were significant, $p<.05$, see Figure 2A). In contrast, during practice before the switch, the main effect of set size disappeared after seven runs and as early as in run three pairwise comparison between set sizes 8 and 15 were nonsignificant. In the first run the error rate (wrong button presses and missing responses) was 25\% (Figure 2B). The high error rate in run 7 coincided with the decrease in trial time to $800 \mathrm{msec}$. With practice, the error rate decreased to $5 \%$ before the switch, increased postswitch to 18\%, and decreased again over the last 10 runs.

\section{Experiment 2}

Of 72 participants, 49 satisfied the criteria for automaticity. As in Experiment 1, a repeated measures ANOVA 
Table 1. Brodmann's Areas with Significant Activation at Any One Time during Task Performance

\begin{tabular}{|c|c|c|c|c|c|c|}
\hline \multirow[b]{2}{*}{$B A$} & \multirow[b]{2}{*}{ Structure } & \multirow[b]{2}{*}{ Hemisphere } & \multirow[b]{2}{*}{ Volume $(\mu l)$} & \multicolumn{3}{|c|}{$\begin{array}{c}\text { Center of Mass for the Largest } \\
\text { Cluster within the BA (Talairach } \\
\text { and Tourneaux Atlas) }\end{array}$} \\
\hline & & & & $x$ & $y$ & $z$ \\
\hline \multicolumn{7}{|c|}{ Areas in which activation decreased significantly with practice } \\
\hline \multicolumn{7}{|c|}{ Frontal lobe } \\
\hline 6 & Precentral, middle frontal, medial frontal gyri & Bilateral & 15,294 & -37 & -3 & 44 \\
\hline 8 & Medial and superior frontal gyri & $\mathrm{R}>\mathrm{L}$ & 1,126 & 4 & 24 & 45 \\
\hline 9 & Medial and superior frontal gyri & $\mathrm{L}$ & 376 & -4 & 22 & 46 \\
\hline 10 & $\begin{array}{l}\text { Superior, middle, and inferior, frontal gyri, } \\
\text { precentral gyrus }\end{array}$ & Bilateral & 2,880 & 43 & 12 & 32 \\
\hline 13 & Middle frontal gyrus & Bilateral & 249 & -36 & 41 & 20 \\
\hline 24 & Inferior frontal gyrus, insula & $\mathrm{R}>\mathrm{L}$ & 3,201 & 37 & 14 & 7 \\
\hline 32 & Cingulate gyrus & Bilateral & 2,902 & -9 & 4 & 39 \\
\hline 33 & Cingulate gyrus & Bilateral & 3,346 & -8 & 16 & 37 \\
\hline 44 & Parahippocampal gyrus & Bilateral & 479 & -35 & -32 & -14 \\
\hline 45 & Precentral gyrus & $\mathrm{R}$ & 504 & 53 & 10 & 11 \\
\hline 46 & Inferior frontal gyrus & $\mathrm{R}$ & 183 & 41 & 21 & 4 \\
\hline 47 & Middle frontal gyrus & $\mathrm{R}$ & 206 & 42 & 34 & 16 \\
\hline 2 & Inferior frontal gyrus & Bilateral & 1,021 & 42 & 18 & -2 \\
\hline \multicolumn{7}{|c|}{ Parietal lobe } \\
\hline 39 & Postcentral gyrus & $\mathrm{L}$ & 880 & -46 & -26 & 38 \\
\hline \multirow[t]{2}{*}{40} & Angular gyrus, inferior parietal lobule & $\mathrm{L}$ & 260 & 33 & -61 & 37 \\
\hline & Inferior parietal lobule and supramarginal gyrus & $\mathrm{R}<\mathrm{L}$ & 5,726 & -44 & -40 & 43 \\
\hline \multicolumn{7}{|c|}{ Areas in which activation did not change significantly with practice } \\
\hline \multicolumn{7}{|c|}{ Frontal lobe } \\
\hline 4 & Precentral gyrus & Bilateral & 1,806 & -42 & -16 & 50 \\
\hline 31 & Parahippocampal gyrus & $\mathrm{L}$ & 276 & -9 & -11 & 46 \\
\hline 37 & Fusiform and parahippocampal gyri & $\mathrm{R}<\mathrm{L}$ & 2,976 & 33 & -48 & -11 \\
\hline \multicolumn{7}{|c|}{ Parietal lobe } \\
\hline 3 & Postcentral gyrus & $\mathrm{L}$ & 1,254 & -42 & -20 & 49 \\
\hline 17 & Lingual gyrus, cuneus (inferior occipital gyrus) & Bilateral & 1,741 & -17 & -95 & -9 \\
\hline 23 & Cingulate and posterior cingulate gyrus & Bilateral & 549 & -5 & -23 & 27 \\
\hline \multicolumn{7}{|c|}{ Temporal lobe } \\
\hline 20 & Inferior temporal gyrus & Bilateral & 541 & -33 & -38 & -16 \\
\hline 22 & Superior temporal gyrus & $\mathrm{L}$ & 285 & -49 & 9 & -3 \\
\hline 41 & Transverse and superior temporal gyri & $\mathrm{L}$ & 248 & -52 & -24 & 12 \\
\hline \multicolumn{7}{|c|}{ Occipital lobe } \\
\hline 18 & Middle occipital gyrus & Bilateral & 10,228 & -27 & -90 & 2 \\
\hline 19 & Middle occipital gyrus & Bilateral & 5,804 & 41 & -79 & -1 \\
\hline
\end{tabular}




\begin{tabular}{|c|c|c|c|c|c|c|}
\hline \multirow[b]{2}{*}{$B A$} & \multirow[b]{2}{*}{ Structure } & \multirow[b]{2}{*}{ Hemisphere } & \multirow[b]{2}{*}{ Volume $(\mu l)$} & \\
\hline & & & & $x$ & $y$ & $z$ \\
\hline \multicolumn{7}{|c|}{ Areas in which activation changed significantly from pre- to post switch } \\
\hline \multicolumn{7}{|c|}{ Frontal lobe } \\
\hline 8 & Medial and superior frontal gyri $\uparrow$ & $\mathrm{L}$ & 376 & -4 & 22 & 46 \\
\hline 9 & $\begin{array}{l}\text { Superior, middle, and inferior, frontal gyri, } \\
\text { precentral gyrus } \uparrow\end{array}$ & Bilateral & 2,880 & 43 & 12 & 32 \\
\hline 46 & Middle frontal gyrus $\uparrow$ & $\mathrm{R}$ & 206 & 42 & 34 & 16 \\
\hline \multicolumn{7}{|c|}{ Parietal lobe } \\
\hline 3 & Postcentral gyrus $\downarrow$ & $\mathrm{L}$ & 1,254 & -42 & -20 & 49 \\
\hline 39 & Angular gyrus, inferior parietal lobule $\uparrow$ & $\mathrm{L}$ & 260 & 33 & -61 & 37 \\
\hline 40 & $\begin{array}{l}\text { Inferior parietal lobule and supramarginal } \\
\text { gyrus } \uparrow\end{array}$ & $\mathrm{R}$ & 1,549 & 40 & -45 & 43 \\
\hline
\end{tabular}

Temporal lobe Transverse and superior temporal gyri $\downarrow$

L

248

$-52$

$-24$

12

Areas in which activation decreased significantly with practice and changed significantly from pre- to post switch

Frontal lobe

\begin{tabular}{|c|c|c|c|c|c|c|}
\hline 8 & Medial and superior frontal gyri $\uparrow$ & $\mathrm{L}$ & 376 & -4 & 22 & 46 \\
\hline 9 & $\begin{array}{l}\text { Superior, middle, and inferior, frontal gyri, } \\
\text { precentral gyrus } \uparrow\end{array}$ & Bilateral & 2,880 & 43 & 12 & 32 \\
\hline 46 & Middle frontal gyrus $\uparrow$ & $\mathrm{R}$ & 206 & 42 & 34 & 16 \\
\hline \multicolumn{7}{|c|}{ rietal lobe } \\
\hline 39 & Angular gyrus, inferior parietal lobule $\uparrow$ & $\mathrm{L}$ & 260 & 33 & -61 & 37 \\
\hline
\end{tabular}

The table is subdivided by listing first areas in which activation decreased significantly with practice; second, areas that showed no significant changes in activation with practice; third, areas that differed significantly in activation pre- versus postswitch; and fourth, areas that decreased significantly with practice and that differed significantly pre- versus postswitch. Clusters are first sorted by lobe then by Brodmann's area (BA). $\mathrm{R}=$ right; $\mathrm{L}=$ left; $\mathrm{R}>\mathrm{L}=$ more right hemispheric activation $(p<.05) ; \mathrm{R}<\mathrm{L}=$ more left hemispheric activation $(p<.05)$. $\uparrow$ indicates increased, $\downarrow$ decreased activation from pre- to postswitch. Only BAs with a total volume of $<100 \mu$ are listed.

with runs (first run of practice, last run before the switch, and first run after the switch) and set size (8, $15,20)$ as within-subject factors revealed a main effect of run, $F(2,96)=437.5, p<.001$; set size, $F(2,96)=73.9$, $p<.001$; and a significant interaction, $F(2,96)=12.4$, $p<.001$ (Figure 2C). In the first run, all pairwise comparisons between set sizes were significant (all $p<.01)$. Preswitch reaction time for set size 8 equaled that of set size 15 , and reaction time for set size 15 equaled that of set size 20. In contrast to Experiment 1 , reaction time difference between set sizes 8 and 20 remained significant $(p<.01)$. After the switch, however, the set size effect was much more pronounced (all pairwise comparisons significant, $p<.001)$. Reaction time became asymptotic with practice and increased postswitch (Figure 2C). As in Experiment 1, after the first practice block a significant decrease in reaction time and an increase of the error rate can be seen due to the shortened trial time. Again, it is important to note that the set size effect persisted in the final 10 runs of practice postswitch (repeated measures ANOVA with set size as independent variable calculated for each run; all pairwise comparisons between set sizes within runs were highly significant, $p<.001$ ). The error rate in the first run was 32\%, decreasing to $8 \%$ preswitch and increasing to $16 \%$ postswitch (Figure 2D).

\section{Experiment 3}

Fifteen of 24 participants achieved criterial levels of automaticity. 


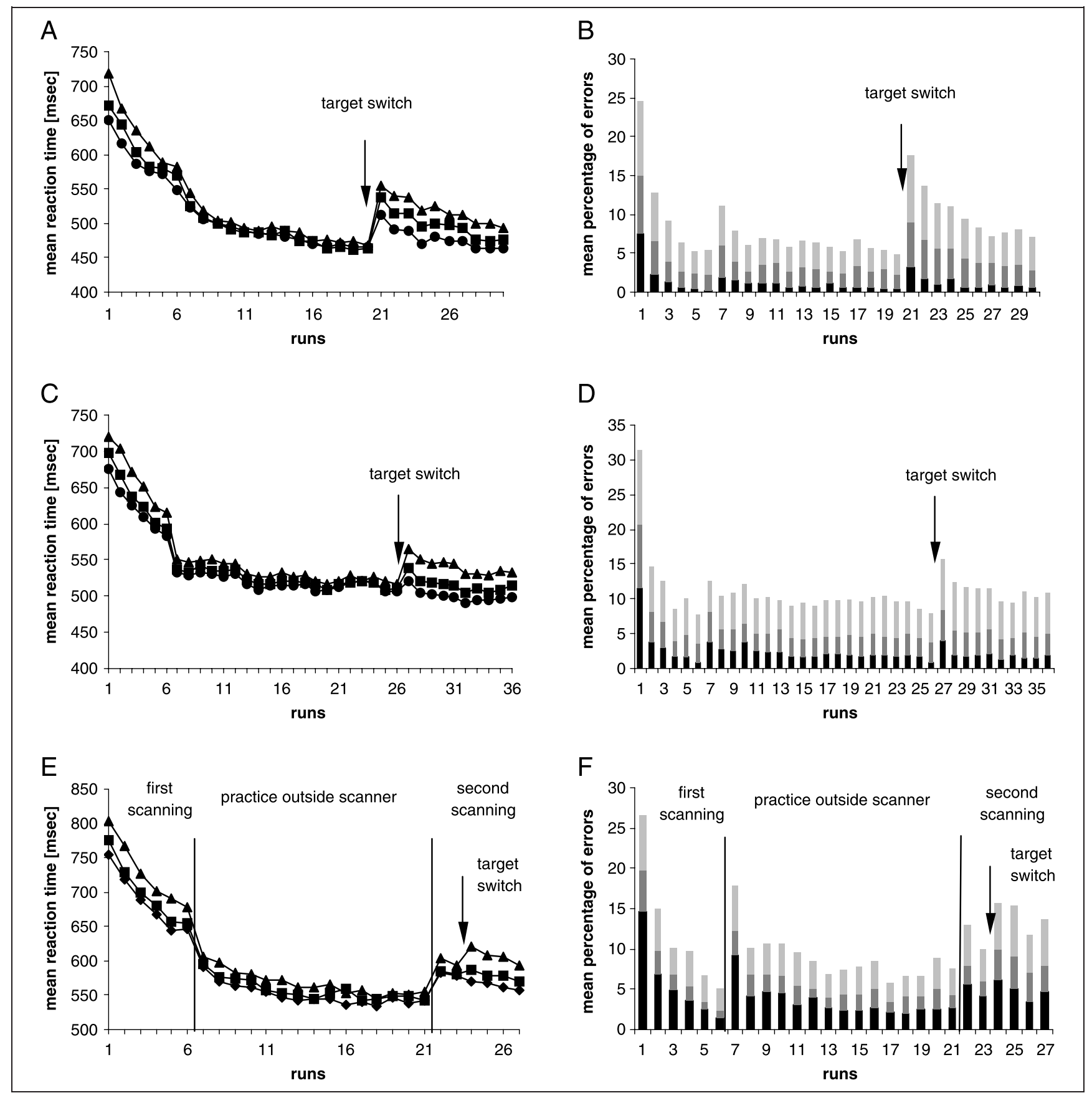

Figure 2. Learning curves (left column) and error rates (right column) for all three experiments (A, B: Experiment 1; C, D: Experiment 2; E, F: Experiment 3). Learning curves: Mean reaction time for each set size is plotted as a function of runs (diamonds: set size 8, cubes: set size 15, triangles: 20). Early in practice and after the target switch the set size effect was clearly visible. With practice, reaction time became asymptotic. After the first practice block of six runs, a pronounced decrease in reaction time occurred in all experiments due to the shortening of the response time window from 1000 to $800 \mathrm{msec}$; accordingly, the error rate increased. In Experiments 1 and 2 , practice continued after the switch and the set size did not disappear within 10 runs. Error rate: mean percentage of errors (black bars: missing responses; dark gray: false positives; light gray: misses). Error rate was high in the first run, decreased with practice, and increased again after the target switch.

\section{Behavioral Data}

As in Experiments 1 and 2, a repeated measures ANOVA with runs (first run of practice, last run before the switch, and first run after the switch) and set size (8, $15,20)$ as within-subject factors found a main effect of run, $F(2,28)=372.3, p<.001$; and of set size, $F(2,28)=$ 38.6, $p<.001$; and a significant interaction, $F(2,96)=$ $3.3, p<.05$. In the first run of practice reaction time differed significantly between all set sizes (all pairwise comparisons, $p<.05$ ). As in Experiment 2, the set size effect did not completely disappear at the end 
of practice (pairwise comparison for set sizes 8 and 20 remained significant, $p<.05$ ). However, after the switch the set size effect reemerged fully with all pairwise comparisons reaching significance $(p<.05$; Figure 2E). After the participants were retransferred into the scanner reaction time increased significantly (repeated measures ANOVA with set size and run as within subject factors, $p<.05$ for all set sizes compared between last run of practice and first run of the second scanning session, Figure $2 \mathrm{E}$ ). The error rate in the first run was $27 \%$, decreasing to $10 \%$ preswitch and increasing to $16 \%$ postswitch (Figure $2 \mathrm{~F}$ ).

\section{Functional Data}

Early in practice we found widespread activation in a fronto-temporo-parietal-occipital network (Figure 3). A repeated measures ANOVA with scans (first scan of practice, last scan before the switch, and first scan after the switch) as within-subject factor was applied to each of 26 functionally defined ROIs. Activation in all frontal areas (BAs 6, 8, 9, 10, 13, 45, 46, and 47) and in some parietal areas (BAs 2, 39, and 40) decreased significantly with practice (pairwise comparisons between Scan 1 and the scan before the switch were significant, $p<.05$ ) (Figures 3, 4A, and 5 and Table 1). In the transverse and superior temporal gyrus (BA 41) activation remained unchanged, whereas in all other areas the pattern was of activation decreases, although pairwise comparisons did not reach significance (Figure 3 and Table 1).

After the switch we found very selective increases in activation in the bilateral dorsolateral prefrontal cortex (DLPFC; BAs 9 and 46; pairwise comparisons of runs preand postswitch were $p<.05$ for BA 9 and $p=.056$ for BA 46) (Figures $4 \mathrm{~b}$ and 5) and in one cluster in BA 8 $(p<.05)$; activation increased also in the angular gyrus and inferior parietal lobule (BA 39 and one cluster in BA $40, p<.01$ ) bilaterally (Figure 5). Activation in the left postcentral gyrus (BA 3) and left transverse and superior temporal gyrus (BA 41) decreased significantly after the switch as compared to before the switch (both $p<.05$ ). In all other areas, we found no difference in activation pre- and postswitch. Repeated measures ANOVA with runs and hemisphere as within-subjects factors found parietal BAs 37 and 40 more activated in the left $(p<.05)$ and frontal BAs 8 and 13 more in the right $(p<.05)$ hemisphere.

\section{DISCUSSION}

With our VST we were able to train participants to criterial levels of automaticity within a reasonable amount of time and to compel them to reestablish executive control over their automatized behavior. Learning curves of all three experiments show the typical logarithmic shape indicating rapid improvement of performance early in practice and asymptotic performance with further practice (Newell \& Rosenbloom, 1981). They also show a prominent set size effect at the beginning of training that reduced dramatically with practice, indicating a transition from serial search to memory retrieval, a hallmark of automatic behavior. In contrast to Experiment 1, the set size effect did not disappear completely in Experiments 2 and 3. We attribute these effects to massed practice, which may have led to concentration problems and fatigue in late practice and which may have provided less time for memory consolidation. Despite the set size effect not disappearing completely, it is very apparent that the set size effect differed substantially between early and late in practice preswitch and also between pre- and postswitch. Noting that automaticity exists to a matter of degree, this renders the brain comparison at the different task stages meaningful. Had the set size effect in Experiment 3 disappeared completely preswitch (as in Experiment 1) we would expect the functional effects to be even more robust.

Widespread activation in the frontal cortex, which comprises generic attention and control areas, decreased considerably with practice, a phenomenon frequently observed in studies examining task practice (e.g., Poldrack et al., 2005; Debaere et al., 2004; Shadmehr \& Holcomb, 1997) (see Kelly \& Garavan, 2005, for a review). Reduced dependence on frontal areas with task practice is in accordance with the notion that automatic processes place greatly reduced attentional demands on an individual. The target switch required the participants to recommence serial search, to inhibit the automatic, prepotent response, and to learn new stimulus-response mappings. As expected, after the target switch the set size effect reappeared in all three experiments. Reaction time increased but was significantly below that observed early in practice indicating a general learning effect that persisted from pre- to postswitch. In Experiments 1 and 2, participants were trained for another 10 runs after the switch. In both experiments, the set size effect remained highly significant across these 10 runs. Conversely, early in practice the set size effect decreased within six to seven runs, indicating that postswitch performance suffered interference from preswitch learning and thus reflected more than the learning of new stimulus-response mappings. The data of Experiment 1 suggest that the exaggerated postswitch set size effect cannot be attributed to fatigue because participants were trained for $30 \mathrm{~min}$ on five consecutive days. It has previously been demonstrated that unlearning an automatic memory search set and learning a new search set takes longer than learning the original search set (Schneider \& Chein, 2003; Shiffrin \& Schneider, 1977). Thus, we interpret this result as evidence that the switch necessitated the reestablishment of executive control over automaticity rather than just the learning of a new task. 
Figure 3. Axial view of eight slices. Scans $1-3$ early in practice, Scan 4 last scan before the switch, Scan 5 first scan after the switch, Scan 6 last scan of experiment. Activation decreased with practice. In few areas such as BA 9, activation increased after the switch.

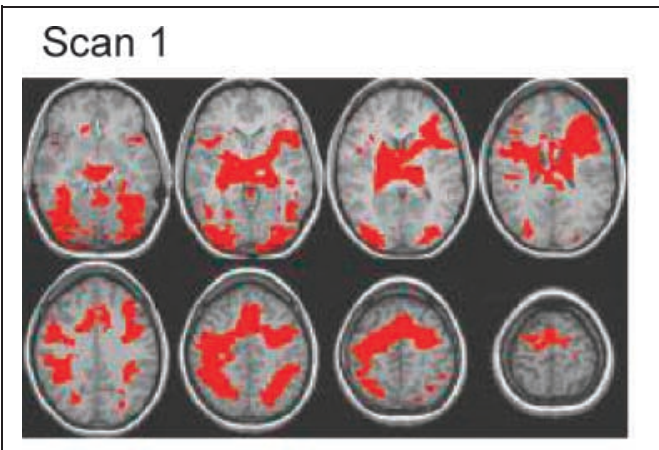

\section{Scan 2}

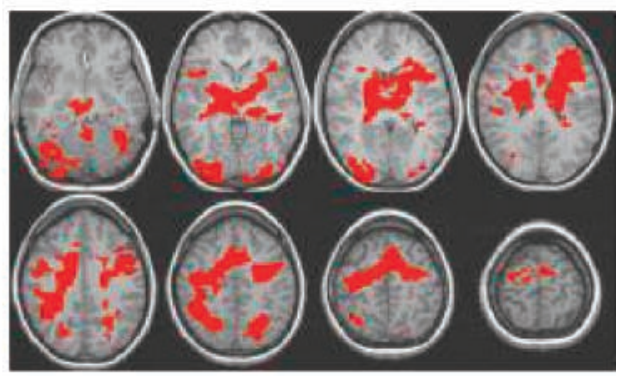

\section{Scan 3}

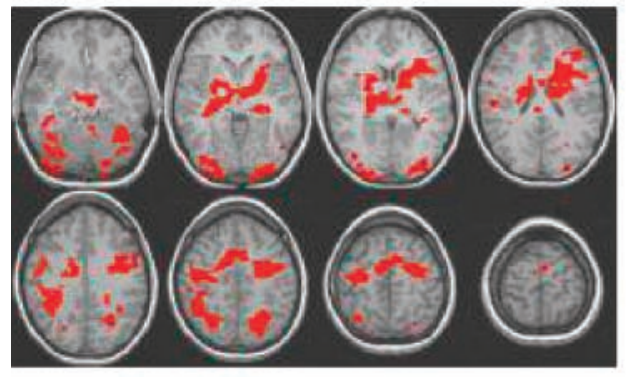

\section{Scan 4}

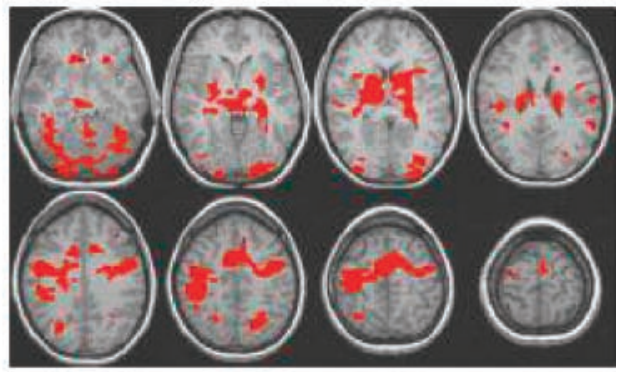

\section{Scan 5}

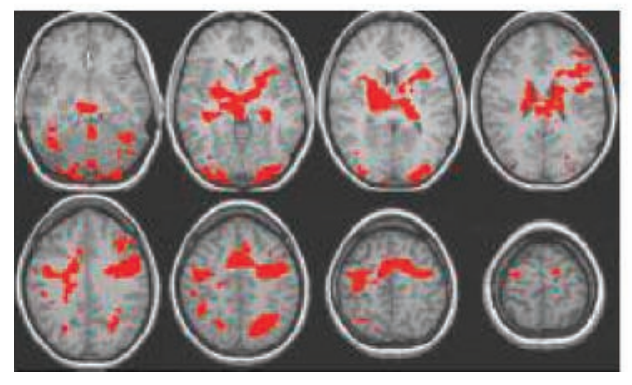

\section{Scan 6}

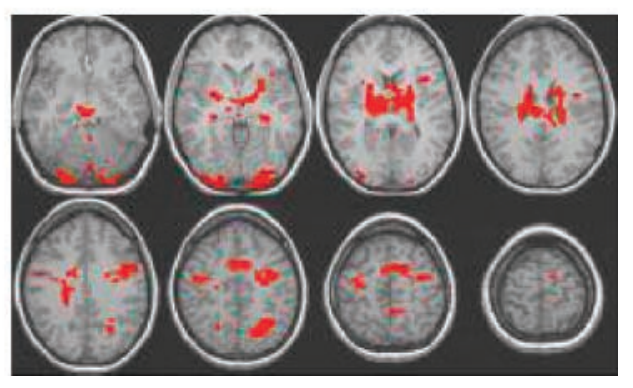

Widespread activation, as was observed at the beginning of practice, was not recapitulated postswitch. Instead, of all the frontal areas activated at the beginning of the task, in all of which activation decreased significantly with practice, only the DLPFC showed increased activation when reestablishment of executive control over automatized behavior was required. The DLPFC is well known to play a crucial role in executive functioning, that is, in controlling overt, deliberate, intentional (nonautomatic) behavior (Fassbender et al., 2004; Collette \& van der Linden, 2002; Wagner, Maril, Bjork, \& Schacter, 2001).
Figure 4. Axial view (A) at $z$ coordinate 41 of frontal and parietal areas that decreased with practice (see Table 1 for peak activation coordinates). Coronal view (B) at $y$ coordinate 6 of activation in DLPFC (BA 9) in which activation increased significantly after the target switch.

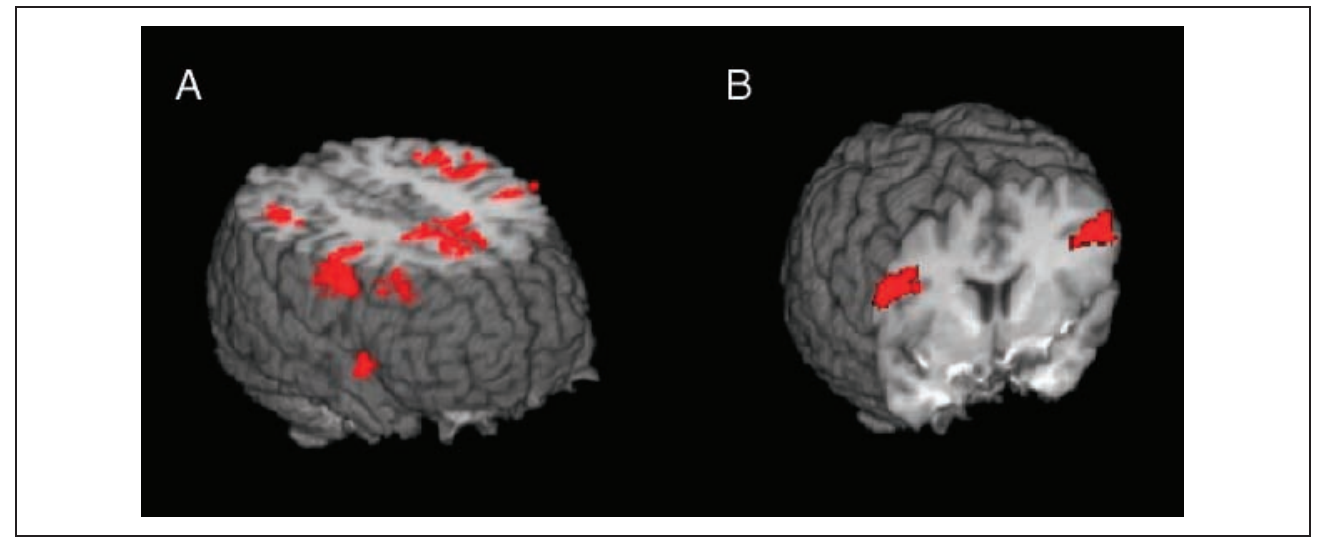




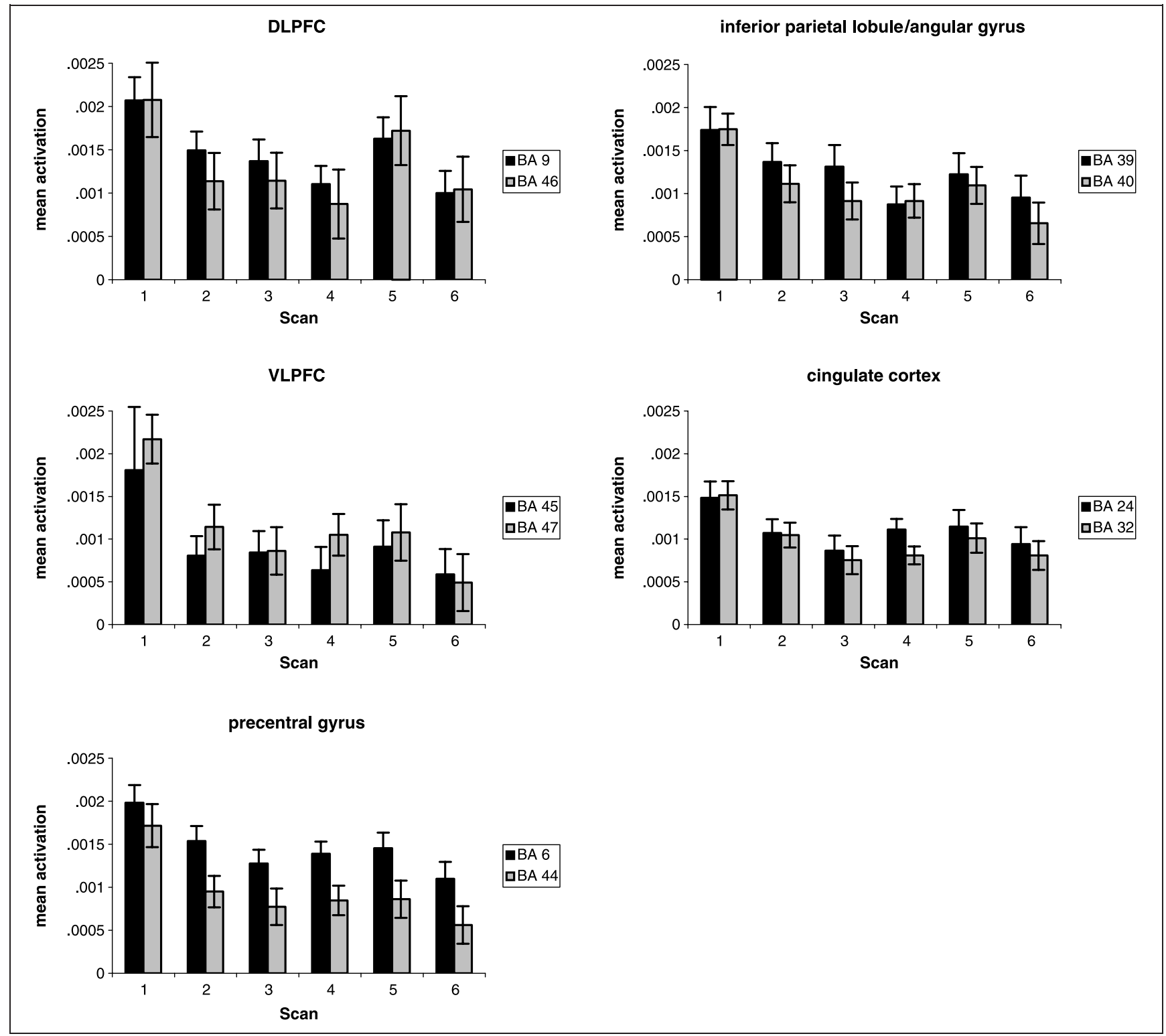

Figure 5. Activation early (Scans 1-3) and late in practice (Scan 4) and after the switch (Scans 5 and 6) in selected BAs. Only in BAs 9, 46, and 39 and in one cluster in BA 40 (and one cluster in BA 8, not shown here) did activation increase significantly after the switch. Error bars represent SEM.

Neurons in the posterior parietal cortex (including BAs 5, 7, 39, and 40) receive combined information from sensory and motor areas. Their firing rate is increased when attention is directed to a target (Stein, 1989). This renders these cells suitable to control attention and motor behavior according to sensory input. Involvement of the parietal lobes in executive functioning is often observed and has been proposed as being necessary for the actual enactment of frontal commands (Kübler, Murphy, Kaufman, et al., 2003). Accordingly, with increasing automaticity, activation decreased in inferior parietal lobule (BAs 39 and 40). We may speculate that the postswitch activation of angular gyrus and inferior parietal lobule (BA 39) reflects an increased attentional response or the creation and establishment of new stimulus-response mappings according to the new task requirements identified by the DLPFC. After the switch the number of missing responses increased, which could account for the decreased activation in the left postcentral gyrus (BA 3).

The dynamics involved in the development of automaticity and, most importantly, in suppressing unwanted automatic behaviors are consequential for many everyday functions as well as being clinically relevant. Although common-day examples may range from turning left to a colleague's office rather than right to the more frequently visited vending machine or to driving on the "other" side of the road when holidaying abroad, there may be many important clinically relevant examples of failures to exert control over habitual behaviors. 
As just one example, compulsive drug use, which has been proposed after many episodes of drug use to adopt the characteristics of automated behavior (Tiffany, 1990), may be regarded as failed executive control over overlearned behavioral habits (Gerdeman, Partridge, Lupica, \& Lovinger, 2003). The specific neuroanatomy found to subserve this executive control may constitute neurocognitive functions that are affected in this population and are thus potential targets for intervention.

\section{Acknowledgments}

The assistance of Alan Jones, Dr. Kevin Murphy, and Dr. Boris Kotchoubey is gratefully acknowledged. This study was supported by NIDA Grant RO1 DA14100.

Reprint requests should be sent to Hugh Garavan, School of Psychology, Trinity College Dublin, Dublin 2, Ireland, or via e-mail: Hugh.Garavan@tcd.ie.

The data reported in this experiment have been deposited with the fMRI Data Center (www.fmridc.org). The accession number is $2-2006-121 \mathrm{BN}$.

\section{Note}

1. The thresholded clusters were required to have a minimum size of $174 \mu \mathrm{l}$. As this can result in clusters that are quite large and that envelop different anatomical regions, we then separated the clusters by Brodmann's areas resulting in clusters that were both functionally and anatomically defined. It is for this region that the clusters entered into the cluster-level statistical analyses were smaller than the $174-\mu$ l criterion. However, in an effort to reduce the number of clusters analyzed within a BA, we restricted the final cluster-level analysis to those Brodmann's areas that contained at least $100 \mu \mathrm{l}$ of activated voxels. In doing so, we excluded from the cluster analyses just $386 \mu$ l of voxels from 12 Brodmann's areas.

\section{REFERENCES}

Beauchamp, M. H., Dagher, A., Aston, J. A., \& Doyon, J. (2003). Dynamic functional changes associated with cognitive skill learning of an adapted version of the Tower of London task. Neuroimage, 20, 1649-1660.

Buchel, C., Coull, J. T., \& Friston, K. J. (1999). The predictive value of changes in effective connectivity for human learning. Science, 283, 1538-1541.

Collette, F., \& van der Linden, M. (2002). Brain imaging of the central executive component of working memory. Neuroscience and Biobehavioral Reviews, 26, 105-125.

Compton, B. J., \& Logan, G. D. (1991). The transition from algorithm to retrieval in memory-based theories of automaticity. Memory \& Cognition, 19, 151-158.

Cox, R. W. (1996). AFNI: Software for analysis and visualization of functional magnetic resonance neuroimages. Computers and Biomedical Research, 29, 162-173.

Debaere, F., Wenderoth, N., Sunaert, S., Van Hecke, P., \& Swinnen, S. P. (2004). Changes in brain activation during the acquisition of a new bimanual coordination task. Neuropsychologia, 42, 855-867.

Fassbender, C., Murphy, K., Foxe, J. J., Wylie, G. R., Javitt, D. C., Robertson, I. H., \& Garavan, H. (2004). A topography of executive functions and their interactions revealed by functional magnetic resonance imaging. Brain Research, Cognitive Brain Research, 20, 132-143.

Frutiger, S. A., Strother, S. C., Anderson, J. R., Sidtis, J. J., Arnold, J. B., \& Rottenberg, D. A. (2000). Multivariate predictive relationship between kinematic and functional activation patterns in a PET study of visuomotor learning Neuroimage, 12, 515-527.

Gerdeman, G. L., Partridge, J. G., Lupica, C. R., \& Lovinger, D. M. (2003). It could be habit forming: Drugs of abuse and striatal synaptic plasticity. Trends in Neurosciences, 26, 184-192.

Hester, R., Foxe, J. J., Molholm, S., Shpaner, M., \& Garavan, H. (2005). Neural mechanisms involved in error processing: A comparison of errors made with and without awareness. Neuroimage, 27, 602-608.

Holm, S. A. (1979). Simple sequentially rejective multiple test procedure. Scandinavian Journal of Statistics, 6, 65-70.

Jueptner, M., Stephan, K. M., Frith, C. D., Brooks, D. J., Frackowiak, R. S. J., \& Passingham, R. E. (1997). Anatomy of motor learning. 1. Frontal cortex and attention to action. Journal of Neurophysiology, 77, 1313-1324.

Kaufman, J. N., Ross, T. J., Stein, E. A., \& Garavan, H. (2003). Cingulate hypoactivity in cocaine users during a GO-NOGO task as revealed by event-related functional magnetic resonance imaging. Journal of Neuroscience, 23, 7839-7843.

Kelly, A. M., \& Garavan, H. (2005). Human functional neuroimaging of brain changes associated with practice. Cerebral Cortex, 15, 1089-1102.

Klapp, S. T., Boches, C. A., Trabert, M. L., \& Logan, G. D. (1991). Automatizing alphabet arithmetic: II. Are there practice effects after automaticity is achieved? Journal of Experimental Psychology: Learning, Memory, and Cognition, 17, 196-209.

Kübler, A., Murphy, K., \& Garavan, H. (2005). Cocaine dependence and attention switching within and between verbal and visuospatial working memory. European Journal of Neuroscience, 2, 1984-1992.

Kübler, A., Murphy, K., Kaufman, J., Stein, E. A., \& Garavan, H. (2003). Co-ordination within and between verbal and visuospatial working memory: Network modulation and anterior frontal recruitment. Neuroimage, 20, 1298-1308.

Logan, G. D. (1988). Toward an instance theory of automatization. Psychological Review, 95, 492-527.

Logan, G. D. (2002). An instance theory of attention and memory. Psychological Review, 109, 376-400.

Logan, G. D., \& Klapp, S. T. (1991). Automatizing alphabet arithmetic: I. Is extended practice necessary to produce automaticity? Journal of Experimental Psychology: Learning, Memory, and Cognition, 17, 179-195.

Newell, A., \& Rosenbloom, P. S. (1981). Mechanisms of skill acquisition and the law of practice. In J. R. Anderson (Ed.), Cognitive skills and their acquisition (pp. 1-55). Hillsdale, NJ: Erlbaum.

Palmeri, T. J. (1997). Exemplar similarity and the development of automaticity. Journal of Experimental Psychology: Learning, Memory, and Cognition, 23, 324-354.

Poldrack, R. A., \& Gabrieli, J. D. E. (2001). Characterizing the neural mechanisms of skill learning and repetition priming: Evidence from mirror reading. Brain, 124, 67-82.

Poldrack, R. A., Sabb, F. W., Foerde, K., Tom, S. M., Asarnow, R. F., Bookheimer, S. Y., \& Knowlton, B. J. (2005). The neural correlates of motor skill automaticity. Journal of Neuroscience, 25, 5356-5364.

Raichle, M. E., Fiez, J. A., Videen, T. O., MacLeod, A. M., Pardo, J. V., Fox, P. T., \& Petersen, S. E. (1994). Practice-related changes in human brain functional anatomy during nonmotor learning. Cerebral Cortex, 4, 8-26. 
Schneider, W., \& Chein, J. M. (2003). Controlled and automatic processing: Behavior, theory, and biological mechanisms. Cognitive Science, 27, 525-559.

Schneider, W., \& Shiffrin, R. M. (1977). Controlled and automatic human information processing: I. Detection, search and attention. Psychological Review, 84, 1-190.

Shadmehr, R., \& Holcomb, H. H. (1997). Neural correlates of motor memory consolidation. Science, 277, 821-825.

Shiffrin, R. M., \& Schneider, W. (1977). Controlled and automatic human information processing: II. Perceptual learning, automatic attending, and a general theory. Psychological Review, 84, 127-190.

Stein, J. F. (1989). Representation of egocentric space in the posterior parietal cortex. Quarterly Journal of Experimental Physiology, 74, 583-606.

Stroop, J. R. (1935). Studies of interference in serial verbal reactions. Journal of Experimental Psychology, 18, 643-662.
Talairach, J., \& Tourneaux, P. (1988). Co-planar stereotaxic atlas of the buman brain. New York: Thieme.

Tiffany, S. T. (1990). A cognitive model of drug urges and drug-use behavior: Role of automatic and nonautomatic processes. Psychological Review, 97, 147-168.

Toni, I., Rowe, J., Stephan, K. E., \& Passingham, R. E. (2002). Changes of cortico-striatal effective connectivity during visuomotor learning. Cerebral Cortex, 12, 1040-1047.

Wagner, A. D., Maril, A., Bjork, R. A., \& Schacter, D. L. (2001). Prefrontal contributions to executive control: fMRI evidence for functional distinctions within lateral prefrontal cortex. Neuroimage, 14, 1337-1347.

Wong, E. C., Boskamp, E., \& Hyde, J. S. (1992). A volume optimized quadrature elliptical endcap birdcage brain coil. Paper presented at the Eleventh Annual Scientific Meeting, Society for Magnetic Resonance Medicine, Berlin. 